\title{
Passive component based multifunctional Jones matrix swept source optical coherence tomography for Doppler and polarization imaging
}

\author{
Yiheng Lim, Young-Joo Hong, Lian Duan, Masahiro Yamanari, and Yoshiaki Yasuno* \\ Computational Optics Group, University of Tsukuba, Tsukuba, Ibaraki, Japan \\ *Corresponding author: yasuno@optlab2.bk.tsukuba.ac.jp
}

Received January 18, 2012; revised April 4, 2012; accepted April 6, 2012; posted April 9, 2012 (Doc. ID 161758); published May 25, 2012

\begin{abstract}
We present a fiber based multifunctional Jones matrix swept source optical coherence tomography (SS-OCT) system for Doppler and polarization imaging. Jones matrix measurement without using active components such as electrooptic modulators is realized by incident polarization multiplexing based on independent delay of two orthogonal polarization states and polarization diversity detection. In addition to polarization sensitivity, this system measures Doppler flow without extra hardware for phase stabilized SS-OCT detection. An eighth-wave plate was measured to demonstrate the polarization detection accuracy. The optic nerve head of a retina was measured in vivo. Detailed vasculature and birefringent structures were investigated simultaneously. (c) 2012 Optical Society of America OCIS codes: $170.4500,260.5430,170.3340$.
\end{abstract}

Optical coherence tomography (OCT) is a well-known interferometric technique that has been widely used to image high-resolution cross sections of biological tissues in ophthalmology because of its advantages, such as noninvasive and noncontact measurement.

Functional extensions of OCT, such as polarization sensitive OCT (PS-OCT) [1] and Doppler OCT [2], have been developed to enhance the contrast in the tissues. For instance, PS-OCT demonstrated tissue discrimination imaging [3]. Doppler OCT has been used to image depth-resolved flow in the retina. Visualization of flow and vasculature has importance in the diagnosis of eye diseases [4] including glaucoma and age related macular degeneration.

Recently, several functionally extended OCTs have been demonstrated based on swept source OCT (SSOCT) $[5,6]$. This is partially because of the advantages of SS-OCT over spectral domain OCT, including improved sensitivity with greater imaging depth, $k$-linear sampling, and balanced detection [7].

Considering the advantages of optical fibers, such as system alignment and handling, many functional OCT systems have been developed with the use of fiber optics. However, for PS-OCT, at least two polarization states of the incident light are required to measure the Jones matrix of a sample [ $[$ ] because of the dynamic birefringence in the single-mode fiber of the sample arm. For this purpose, Jones matrix PS-OCT systems with polarization modulators have been demonstrated $[\underline{6}, \underline{8}]$.

Because the polarization modulator is cost ineffective and difficult to stably handle, a fiber based Jones matrix OCT system without the polarization modulator has drawn increasing interest. The purpose of this paper is to demonstrate a fiber based multifunctional swept-source OCT (MF-OCT) system with the implementation of Doppler flow detection and a Jones matrix detection scheme without a polarization modulator.

As shown in Fig. 1, the MF-OCT is based on a fiberbased Mach-Zehnder interferometer consisting of singlemode fiber (HI 1060, Corning, New York). A $100 \mathrm{KHz}$ wavelength sweeping laser (Axsun Technologies Inc.,
Massachusetts), which sweeps over a spectral range of $109 \mathrm{~nm}$ around a center wavelength of $1.06 \mu \mathrm{m}$, was utilized. In the sample arm, a polarizer and a polarization beam splitter are used to split the light into two mutually orthogonal linear polarization states. Both states are reflected by Dove prisms and propagate in two different optical paths, where the Dove prisms are utilized as compact retro-reflectors with beam displacement. By translating one of the Dove prisms, the delay between the two incident polarization states can be arbitrarily controlled. Because of the compact design, the path length drift between the two polarization states caused by temperature fluctuation is negligible. The two polarization states with different delays are recombined by a second polarization beam splitter and coupled into a fiber coupler. The light traveled into the fiber coupler is then focused on the sample. The power of the probe beam was adjusted to be $1.6 \mathrm{~mW}$ at the cornea, which is lower than the ANSI standard of safe exposure limit. The backscattered light from the sample is collected and directed into a polarization diversity detection arm. In the reference arm, two fiber collimators are used to collimate and couple the light into a fiber that is connected to the detection arm. The optical path length of the reference arm is controlled by translation of the fiber collimator, which is used to couple the beam.

In the detection arm, the light beams from the sample arm and the reference arm are recombined using a

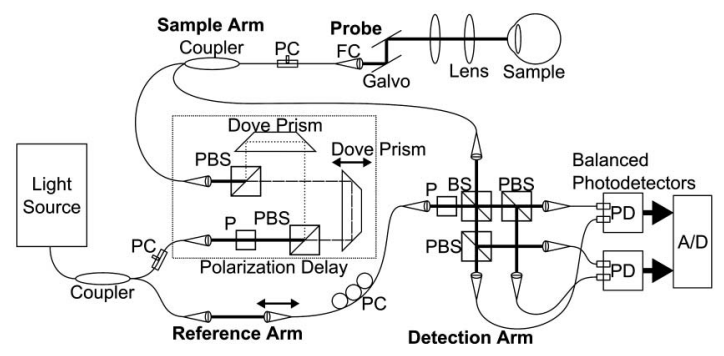

Fig. 1. Scheme of the MF-OCT. A/D, Analog-to-digital converter; BS, beam splitter; FC, fiber collimator; PBS, polarization beam splitter; PC, polarization controller; P, polarizer. 
non-polarization beam splitter (BS). This combined light is then split into two orthogonal states of polarization by two polarization beam splitters (PBS). The interferograms of the two polarization states are detected using two balanced photodetectors (PDB430C, Thorlabs, New Jersey). Prior to the BS, a polarizer is used to ensure equal reference power after the PBSs, as described in Eq. (3) below.

The implementation of the polarization detection can be described using Jones calculus. In the sample arm, the separation of the two orthogonal polarization states into different optical path lengths can be written as

$$
\boldsymbol{E}_{0}=\left[\begin{array}{l}
1 \\
0
\end{array}\right]+\left[\begin{array}{c}
0 \\
e^{i k \zeta}
\end{array}\right]=\left[\begin{array}{c}
1 \\
e^{i k \zeta}
\end{array}\right]
$$

where $k$ and $\zeta$ are the wavenumber of the light source and the relative optical path difference, respectively. Considering the birefringence of the fiber, the Jones matrix at the detection arm can be expressed by using the Jones matrix from the point where the optical path of the orthogonal polarization states is separated to the sample surface $J_{\text {in }}$, a round-trip Jones matrix of the sample $J_{\text {sample }}$, and the Jones matrix from the sample surface to the detection arm $J_{\text {out }}$ :

$$
J_{\text {measured }}=J_{\text {out }} J_{\text {sample }} J_{\text {in }} \equiv\left[\begin{array}{ll}
J_{11} & J_{12} \\
J_{21} & J_{22}
\end{array}\right] .
$$

In the detection arm, the respective electric fields of the sample arm and the reference arm are expressed as

$$
\left\{\begin{array}{c}
\boldsymbol{E}_{\mathrm{samp}}=E_{S} J_{\text {measured }} \boldsymbol{E}_{0} e^{i k z_{\mathrm{samp}}} \\
\boldsymbol{E}_{\mathrm{ref}}=E_{R}\left[\begin{array}{l}
1 \\
1
\end{array}\right] e^{i k z_{\mathrm{ref}}},
\end{array}\right.
$$

where $z_{\text {samp }}$ and $z_{\text {ref }}$ are the path lengths of the sample and reference arms, and $E_{S}$ and $E_{R}$ are scalar constants that define the amplitudes of the sample and reference beams.

In the detection arm, the two beams were split into two orthogonal polarization states. The interferogram of the polarization state, denoted as the horizontal state, is the square of the summation of the horizontal elements of $\boldsymbol{E}_{\text {samp }}$ and $\boldsymbol{E}_{\text {ref }}$ of Eq. (3):

$$
\left\{\begin{array}{c}
E_{\mathrm{samp}, h}=E_{S}\left(J_{11}+J_{12} e^{i k \zeta}\right) e^{i k z_{\mathrm{samp}}} \\
E_{\mathrm{ref}, h}=E_{R} e^{i k z_{\mathrm{ref}}}
\end{array} .\right.
$$

The detected current from the balanced photodetector at the corresponding state is given by $I_{h}(\Delta z) \propto \mid E_{\text {samp }, h}+$ $\left.E_{\text {ref }, h}\right|^{2} \approx J_{11} e^{i k \Delta z}+J_{12} e^{i k(\Delta z+\zeta)}+$ c.c., where common modes are omitted because it is rejected by balanced detection, c.c. denotes the complex conjugate of the terms that are associated with $J_{11}$ and $J_{12}$, and $\Delta z=$ $z_{\text {samp }}-z_{\text {ref }}$. A discrete Fourier transform of this signal yields an OCT signal of

$$
\tilde{I}_{h}(\Delta Z) \propto J_{11} \cdot \delta(z-\Delta z)+J_{12} \cdot \delta(z-(\Delta z+\zeta)) .
$$

Because $J_{11}$ and $J_{12}$ are separated by a distance $\zeta$, the process above has provided us these two of the elements of $J_{\text {measured }}$ in a depth-resolved manner. Similarly, $J_{21}$ and $J_{22}$ can be obtained from the photocurrent of the vertical polarization detector as

$$
\tilde{I}_{v}(\Delta Z) \propto J_{21} \cdot \delta(z-\Delta z)+J_{22} \cdot \delta(z-(\Delta z+\zeta)) .
$$

Because the measured Jones matrix consists of the birefringence of the fiber, it must be cancelled. The Jones matrix of the sample surface $J_{\text {surf }}=J_{\text {out }} J_{\text {in }}$ is extracted, inverted, and then multiplied by the measured Jones matrix to cancel the birefringence of the fiber $[\underline{6}, \underline{8}]$ as $J_{c}=J_{\text {measured }}\left(J_{\text {out }} J_{\text {in }}\right)^{-1}=J_{\text {out }}^{-1} J_{\text {sample }} J_{\text {out }}$, where the fiber is assumed to be lossless, and whose Jones matrix is a unitary matrix. This assumption allows us to obtain the birefringence of the sample by decomposing the Jones matrix obtained into a diagonal matrix $D$ and a unitary matrix $U$, as $J_{c}=U^{-1} D U$. The diagonal elements of $D, P_{1} \exp (i \eta / 2)$ and $P_{2} \exp (-i \eta / 2)$ provide a double pass phase retardation of the sample $\eta$, and the diattenuation coefficients, $P_{1}$ and $P_{2}$. The relative orientation of the optical axis can be calculated from $U$.

Along with the polarization detection, Doppler flow detection is achieved simultaneously using a phase sensitive Doppler method similar to that in [9], in which the Doppler shift is obtained from the phase difference between the adjacent depth-scans. The power of the Doppler shift $\Delta \varphi^{2}$ is used for visualization of the blood vessels [10]. Because the MF-OCT measures the Jones matrix of the sample, the calculation of $\Delta \varphi^{2}$ is applied to each element of the matrix, and the average of the result is used for the Doppler visualization.

Because the polarization measurement and the Doppler algorithms of this MF-OCT system are phase sensitive, it is problematic having phase error in the measurement that is caused by the jitter in the sweeping trigger of the SS-OCT and the bulk motion of the sample. This phase error was canceled using a numerical phase stabilization method presented in [9].

In our specific implementation of this MF-OCT system, the axial resolution was measured to be $9.0 \mu \mathrm{m}$ in air. The sensitivity of system was $85 \mathrm{~dB}$, the roll-off was $-0.76 \mathrm{~dB} / \mathrm{mm}$, and the depth imaging range was $1.89 \mathrm{~mm}$. Although the roll-off results in the difference in sensitivities between two depth-encoded polarization channels of around $1.4 \mathrm{~dB}$, this effect is canceled in the formerly mentioned fiber birefringence cancelation process and does not harm the phase retardation measurement. Since this system relies on depth encoded polarization multiplexing,

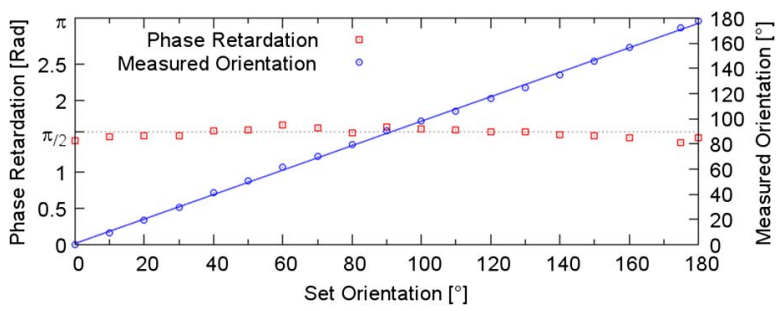

Fig. 2. (Color online) Measured phase retardation, and relative orientation of an eighth-wave plate. 


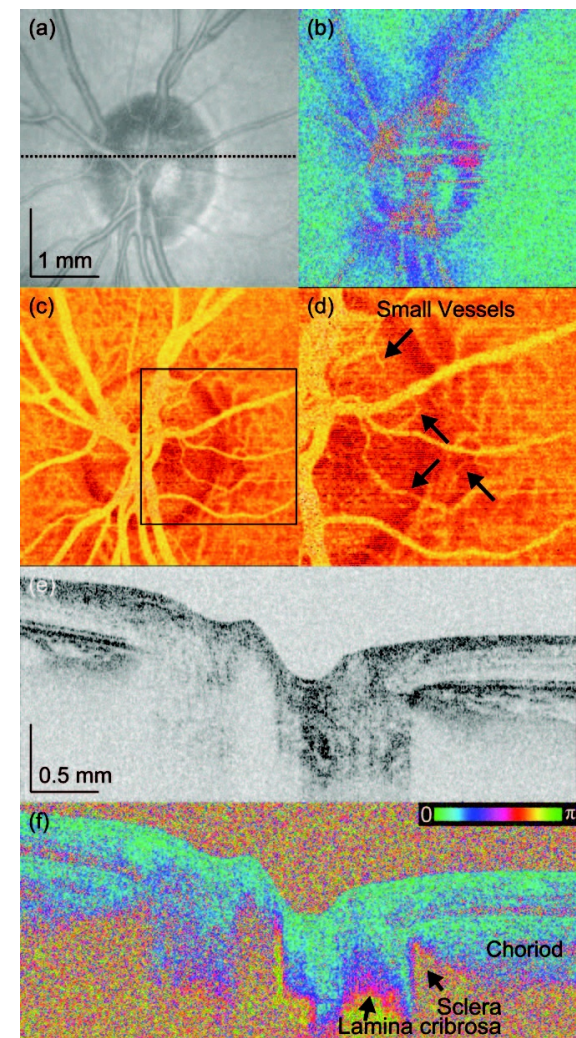

Fig. 3. En face images of (a) intensity, (b) phase retardation, and (c) power of Doppler shift, of an optic nerve head. (d) Magnified image of the square area in (c). (e) Intensity and (f) phase retardation cross section taken from the dashed line in (a).

the depth measurement range is relatively short. This issue could be overcome by using denser spectral sampling and a light source with higher coherence length.

An eighth-wave plate was measured to quantify the Jones matrix measurement capability. Figure 2 shows the measurement of the double pass phase retardation and the relative axis orientation of the wave plate as it was rotated from $0^{\circ}$ to $180^{\circ}$. The double pass phase retardation was measured to be $88.5 \pm 3.7^{\circ}$. The measured orientation was fitted to a linear regression line, and the slope became 0.97 with $R^{2}=1.00$.

Volumetric in vivo measurement was performed on a retina without marked posterior disorder, where $1 \mathrm{~B}$-scan consists of 512 depth-scans and 1 volume consists of 1024 B-scans. Four B-scans were acquired at a single location to apply Jones matrix averaging [11]. Figure 3 shows the en face images of intensity (a), phase retardation measured at a retinal pigment epithelium (b) and $\Delta \varphi^{2}$ (c) obtained from a single measurement of the optic nerve head $(\mathrm{ONH})$. Figure 3(e) is the intensity average of 4 elements of a Jones matrix, and Fig. 3(f) has been obtained from averaged Jones matrix. Figure $3(\mathrm{~d})$ shows a magnified image of the area indicated by a black box in 3(c). Figures $3(\mathrm{e})$ and (f) are the cross sections of the intensity and the phase retardation at the horizontal line in Fig. $3(\mathrm{a})$.
The structure of the $\mathrm{ONH}$ was visualized in the intensity images, Figs. 3(a) and (e), and large vessels were observed in the en face image. In $\Delta \varphi^{2}$ images, Figs. 3(c) and (d), detailed vasculature can be seen. Small vessels, indicated by arrows, were clearly observed. The phase retardation image, Fig. 3(b), shows a bow-tie pattern, which can also be observed using the previously developed PS-OCT system [12]. In the phase retardation cross section [Fig. 3(f)], high phase retardation appears in the tissues that consist of collagen fibers, i.e., the sclera and the lamina cribrosa. Contrast for these fibrous tissues was evidently enhanced by the phase retardation image.

The concept of multiplexing polarization channels into different depth position has also been utilized in recently published unpolarized light based PS-OCT [13]. In comparison to our method, the requirement of low signal rolloff is less, while it requires active frequency shifters.

In conclusion, an MF-OCT was implemented with Doppler detection and a polarization sensitive detect scheme. Besides visualizing the structure of a sample by an intensity image, polarization detection enhances the contrast of the birefringent tissues, and Doppler flow detection allows visualization of the blood vessels. The MF-OCT shows promising potential capabilities for extension of the clinical applications of OCT.

\section{References}

1. C. E. Saxer, J. F. de Boer, B. H. Park, Y. Zhao, Z. Chen, and J. S. Nelson, Opt. Lett. 25, 1355 (2000).

2. Y. Zhao, Z. Chen, C. Saxer, S. Xiang, J. F. de Boer, and J. S. Nelson, Opt. Lett. 25, 114 (2000).

3. A. Miyazawa, M. Yamanari, S. Makita, M. Miura, K. Kawana, K. Iwaya, H. Goto, and Y. Yasuno, Opt. Express 17, 17426 (2009).

4. J. Flammer, S. Orgul, V.P. Costa, N. Orzalesi, G. K. Krieglstein, L. M. Serra, J.-P. Renard, and E. Stefansson, Prog. Retin. Eye Res. 21, 359 (2002).

5. B. J. Vakoc, R. M. Lanning, J. A. Tyrrell, T. P. Padera, L. A. Bartlett, T. Stylianopoulos, L. L. Munn, G. J. Tearney, D. Fukumura, R. K. Jain, and B. E. Bouma, Nat. Med. 15, 1219 (2009).

6. M. Yamanari, S. Makita, and Y. Yasuno, Opt. Express 16, 5892 (2008).

7. B. Potsaid, B. Baumann, D. Huang, S. Barry, A. E. Cable, J. S. Schuman, J. S. Duker, and J. G. Fujimoto, Opt. Express 18, 20029 (2010).

8. B. H. Park, M. C. Pierce, B. Cense, and J. F. de Boer, Opt. Lett. 29, 2512 (2004).

9. Y. Hong, S. Makita, F. Jaillon, M. J. Ju, E. J. Min, B. H. Lee, M. Itoh, M. Miura, and Y. Yasuno, Opt. Express 20, 2740 (2012).

10. S. Makita, Y. Hong, M. Yamanari, T. Yatagai, and Y. Yasuno, Opt. Express 14, 7821 (2006).

11. Y. Lim, M. Yamanari, S. Fukuda, Y. Kaji, T. Kiuchi, M. Miura, T. Oshika, and Y. Yasuno, Biomed. Opt. Express 2, 2392 (2011).

12. M. Yamanari, M. Miura, S. Makita, T. Yatagai, and Y. Yasuno, J. Biomed. Opt. 13, 014013 (2008).

13. K. H. Kim, B. H. Park, Y. Tu, Y. Hasan, B. L. Lee, J. Li, and J. F. de Boer, Opt. Express 19, 552 (2011). 